\title{
Rational use of antibiotics in the context of China's health system reform
}

\author{
Despite improvements in antibiotic use in tertiary hospitals, problems remain in other parts of the \\ health system, say Ping He and colleagues
}

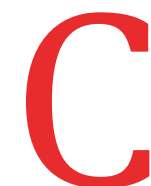

hina spends a high proportion of its total health expenditure on drugs. ${ }^{1}$ In 2006 , almost half $(42.7 \%)$ of the total expenditure on health was on medicines. ${ }^{2}$ It has a serious problem with irrational use of medicines-that is, medically inappropriate or ineffective drug treatment practices. ${ }^{3}$ The main types of irrational use are polypharmacy, misuse of antibiotics, overuse of injections, failure to prescribe according to standard treatment guidelines, and inappropriate self medication. ${ }^{4}$ It is estimated that around half of antibiotics are unnecessarily prescribed. ${ }^{5}$ Overuse and misuse of antibiotics is one of the key contributors to antimicrobial resistance. ${ }^{6}$

Perverse financial incentives for healthcare facilities and physicians have been widely recognised as the major cause of overprescription of antibiotics. ${ }^{7}$ In the late 1970s, China's market oriented economic reform reduced the role of the government in financing healthcare services. ${ }^{8}$ To compensate for the reduced subsidy, the government officially allowed a $15 \%$ or more mark-up on medicines for health facilities. ${ }^{9}$ Since a bonus system

\section{KEY MESSAGES}

- Since the 2009 health system reform, China has paid considerable attention to antibiotic control through strengthening national antimicrobial stewardship and establishing the National Essential Medicines System

- While a national campaign of antimicrobial stewardship was effective in reducing the consumption of antimicrobial agents at tertiary hospitals, the drug policy reform did not improve current problems of antibiotic overuse in primary care and rural settings

- Improving rational use of antibiotics requires not only cross sector coordination and comprehensive intervention but also participation of multiple stakeholders, particularly active engagement from healthcare providers and patients rewarded physicians based on the monetary values of drugs they prescribed, physicians had a strong financial incentive to overprescribe and oversell antibiotics. ${ }^{10}$ The high frequency of irrational use of antibiotics is also exacerbated by decades of misperception that antibacterial medicines are the panacea for all infections. $^{11}$

China embarked on a comprehensive health system reform in 2009. Improving the rational use of antibiotics was a key objective of the national essential medicines system, which was one of five major policies in the 2009 reform. ${ }^{12}$ The policy reform intended to curb the use of antibiotics by unlinking the financial relation between drug prescription and physician income, and by reducing the rate of antimicrobial resistance through comprehensive stewardship of prescribed antibiotics. This paper looks at antibiotic use after the reform and considers how to make further progress on rational use of antibiotics.

\section{Major reform policies}

Since the 2009 health system reform, the Chinese government has been committed to tackling the irrational use of antibiotics by enhancing antimicrobial stewardship. A series of regulations and clinical guidelines for rational use of antibiotics were issued after the reform, and a comprehensive surveillance network including over 1000 member hospitals was built up to control antimicrobial use and resistance. ${ }^{13}$

In 2011, the Ministry of Health launched a national campaign for the rational use of antibiotics, ${ }^{14}$ and in 2012 it enacted and implemented a decree to regulate the clinical use of antibacterial agents. Based on an internationally recognised model of antimicrobial stewardship, this decree included comprehensive regulations on selection, procurement, prescription, and use of antibiotics. The decree also put emphasis on monitoring to ensure the enforcement of these regulations, so it is recognised as the most exacting decree for antibiotic management in China. ${ }^{1516}$ In the same year, the Ministry of Health updated the national guidelines for antibiotic use in clinical practice for public hospitals and mandated hospitals to regularly review and evaluate antibiotic prescriptions of these hospitals. ${ }^{17}$ The national guidelines for antimicrobial therapy implemented in 2012 were revised in $2017 .{ }^{18}$ Meanwhile, appropriate antibiotic use and the bacterial resistance surveillance network, which was established in 2005, have been greatly enhanced owing to a rapid increase in the number of member hospitals since 2011. ${ }^{18}$

Additionally, as one of five key elements of the health system reform, the national essential medicines system focused on compiling a generic essential medicines list, prioritising rational use of drugs by regulating the delivery, distribution, and reimbursement of these drugs. The zero mark-up drug policy removed the profit margin from drug sales and aimed to control overprescription practices by separating the profit of physicians from drug prescription. The policy was initially piloted at primary care institutions in 2009 and then expanded to county hospitals in 2012. ${ }^{19}$ To support the implementation of the national essential medicines system, a centralised bidding procurement system for drugs was established at provincial level, in which most western medicines at public health institutions were traded and registered in the online system. ${ }^{20}$

\section{Achievements and challenges \\ Reduction in antimicrobial consumption at tertiary hospitals}

After the national campaign for the rational use of antibiotics, studies repeatedly reported a reduction in the overuse of antimicrobial agents at tertiary hospitals. ${ }^{2122}$ The figure shows a decrease in the rate of antibiotic use in both inpatients and outpatients, as well as in the rate of combined use of antibiotics in inpatients at 192 core member tertiary hospitals in the Center of Antibacterial Surveillance. In addition, the intensity of antibiotic use, measured by defined daily dose per patient days, decreased from 2005 to 2017 , especially after the national cam- 


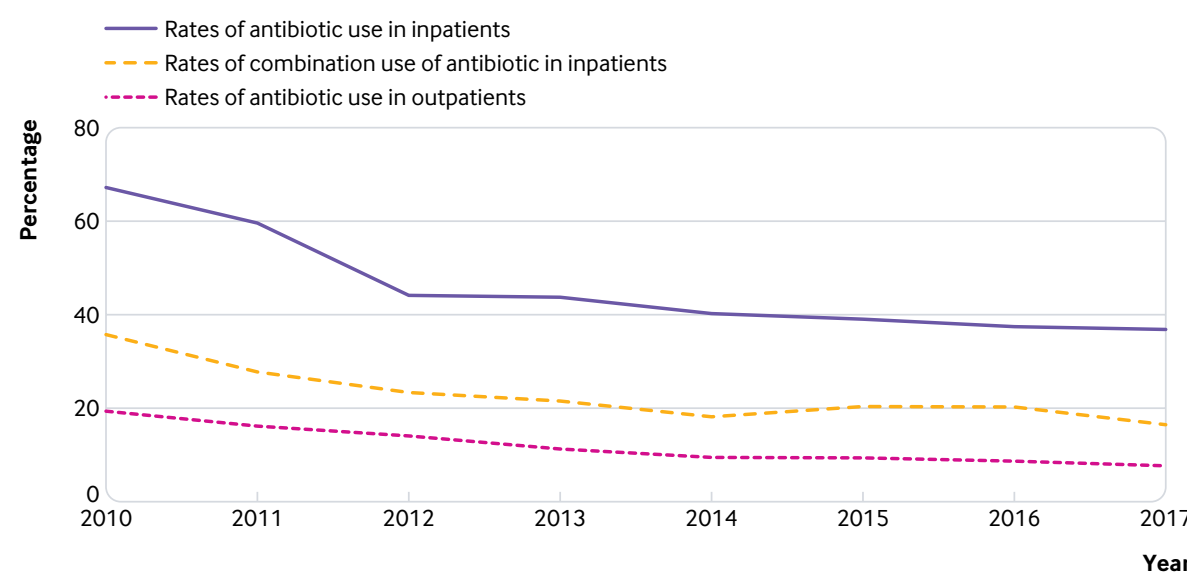

Fig 1 | Trends in rates of antibiotic use in China ${ }^{13}$

paign on the rational use of antibiotics was implemented in 2011. ${ }^{13}$

The rational use of antibiotics campaign, which includes decrees, administrative regulations, clinical guidelines, and drug and incentive policies, built up a regulatory and fiscal framework to curb irrational use of antibiotics. These measures guarantee continuous administrative regulations and the decree has legal effect for compliance or enforcement. For instance, the World Health Organization issued the Notice on the Directory of the Hierarchical Management of Clinical Use of Antimicrobial Agents in 2011. In the same year, a draft version of the Administrative Measures for Clinical Use of Antimicrobial Agents was issued by the legislative affairs office of the State Council, and it was released as Ministry of Health decree 84 in 2012, making it compulsory. The decree includes the general rules, organisations, their duties and responsibilities, management of the clinical use of antibacterial drugs, supervision and oversight, legal liability, and supplementary provisions. After the national campaign, in 2015 the National Health and Family Planning Commission (formerly the Ministry of Health) updated the national guidelines for antibiotic use in clinical practice and set prescription targets for secondary and tertiary hospitals, requiring them to regularly review and evaluate their prescriptions. ${ }^{23}$

\section{Less progress at primary care facilities and county hospitals}

The zero mark-up drug policy was first implemented at primary care institutions and then at public hospitals. Earlier evaluations on primary healthcare facilities found that the policy failed to reduce irrational use of antibiotics in Hubei province. ${ }^{24}$ Further research, using nationwide data on primary care facilities, confirmed a lack of improvement on rational use of antibiotics after the zero mark-up drug policy reform. ${ }^{25}$ Subsequent studies at county hospitals showed similar and consistent results. For example, none of the recent evaluations in Guangxi, ${ }^{26}$ Anhui, ${ }^{27}$ and $\mathrm{Hubei}^{28}$ provinces found improvements on the overuse of antibiotics after the zero mark-up drug policy was implemented at county hospitals.

There are two reasons why the policy did not improve the overuse of antibiotics. Firstly, active physician participation in the interventions is essential to effectively control overuse of antibiotics. In China, the biggest barrier to reducing antibiotic overuse was found to be a lack of qualified physicians, ${ }^{25} 29$ and their poor diagnostic abilities led to unnecessary antibiotic prescriptions at primary care facilities, especially in rural areas. ${ }^{30}$ Furthermore, patients have begun to expect antibiotic treatment. ${ }^{31}$ In addition, after the zero mark-up drug policy reform, the salary and compensation system at county hospitals was not changed, and physicians' income remains dependent on the quantity and revenue of services they provide. ${ }^{4}$ Without changing the profit seeking motivation for public hospitals, piecemeal remedies such as the zero mark-up drug policy will not reduce the overprescription behaviour of providers.

Secondly, patients' knowledge, preference, and demand are important in rational use of antibiotics. Patients consider antibiotics to be a panacea for infections, ${ }^{32}$ leading to willingness to consume antibiotics and exerting pressure on physicians to prescribe more and newer antibiotics. The zero mark-up drug policy theoretically eliminates the drug prescription profit to providers, but it has the potential to increase consumption of antibiotics owing to better affordability for the patient. Therefore, without interventions to change patients' perceptions of the advantages and disadvantages of antibiotic use, the zero mark-up drug policy may not improve overuse of antimicrobials.

\section{Primary care and rural settings have been neglected}

Antimicrobial stewardship during the past decade primarily targeted urban tertiary and secondary hospitals instead of primary care facilities. Despite a decline in antibiotic use at tertiary hospitals, there is little evidence of its improvement in primary care settings. The current rates of antibiotic use are still far higher than the global average and the WHO standard. ${ }^{1} \mathrm{~A}$ systematic review reported that irrational use of antibiotics was more serious in rural areas than in urban areas, as well as more serious at primary care facilities compared with secondary and tertiary hospitals. ${ }^{4}$

\section{Suggestions for making further progress}

Improving rational use of antibiotics requires not only comprehensive interventions, including regulations, health and economic policies, healthcare delivery transformation, and clinical guidelines ${ }^{33}$ but also the participation of multiple stakeholders, in particular the active engagement of both physicians and patients. ${ }^{25} \mathrm{We}$ offer a set of recommendations to improve rational use of antibiotics at the system, provider, and patient level.

Firstly, we recommend that the central government should establish an effective system with cross sector coordination and an integrated surveillance network to improve rational use of antibiotics. The first step is to expand the antimicrobial surveillance network from tertiary and secondary hospitals to all healthcare facilities, and to systematically evaluate the effect on curbing antibiotic use. The second recommended step is to initiate an inter-sector stewardship system on antimicrobials. For example, it is imperative to establish a shared information system between healthcare and agricultural departments to coordinate the monitoring and management of antibiotic use in humans and animals.

Secondly, the policy goal of reducing antibiotic use should be aligned with incentives for healthcare facilities and physicians. One important approach is strengthening the strict implementation of antimicrobial stewardship regulations at hospitals. The most important strategy is eradicating the motivation to overprescribe or overuse antibiotics through increased government subsidies, payment reforms, 
and incentive mechanisms. Motivating physicians to improve rational use of antibiotics is also essential. For physicians at primary care facilities, the recommended approach is to improve their knowledge of antibiotic prescription; and for physicians at hospitals, the optimal approach is to change their profit seeking salary systems.

Thirdly, it is equally important to educate patients, caregivers, and the public on the knowledge and perception of antibiotic use. One intervention is to educate patients and caregivers when they are seeking healthcare services. Another approach is to educate the public about the negative consequences of overuse of antibiotics through the media.

\section{Conclusion}

China's health system reform has made great efforts to reduce the use of antibiotics during the past decade. While a national campaign on antimicrobial stewardship was effective in controlling antibiotic use at tertiary hospitals, the zero mark-up drug policy did not have definite effects at primary care facilities and county hospitals. Further improvement on the irrational use of antibiotics needs a systems approach that integrates the national antimicrobial stewardship network, healthcare providers, and the public.

We thank Dawei Zhu and Xin Ye, from Peking University China Center for Health Development Studies, for their support of document and data analysis; Wen Chen, from Fudan University School of Public Health, for his valuable comments; and Kristina Wharton, from Tulane School of Public Health and Tropical Medicine, for her grammatical editing on the manuscript.

Contributors and sources: PH, QS, LS, and QM'S main research areas are health economics and health policy. PH and QM conceptualised the paper. All authors participated in the study design, data collection, and analysis. PH drafted the paper and all authors contributed to revising the paper.

Competing interests: We have read and understood BMJ policy on declaration of interests and have no relevant interests to declare.

Provenance and peer review: Commissioned; externally peer reviewed.

This article is part of a series proposed by Peking University China Center for Health Development Studies and commissioned by The BMJ. The BMJ retained full editorial control over external peer review, editing, and publication of these articles. Article handling fees (including printing, distribution, and open access fees) are funded by Peking University Health Science Center.

Ping He, assistant professor ${ }^{1}$

Qiang Sun, professor ${ }^{2}$

Lizheng Shi, professor ${ }^{3}$

Qingyue Meng, professor $^{1}$

${ }^{1}$ China Center for Health Development Studies, Peking University, Beijing 100191, China

${ }^{2}$ School of Health Care Management, Shandong

University, Shandong Jinan 250012, China
${ }^{3}$ School of Public Health and Tropical Medicine, Tulane University, New Orleans, LA 70112, USA

Correspondence to: Ping He phe@pku.edu.cn

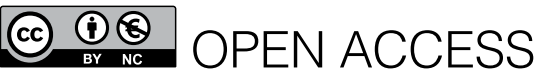

This is an Open Access article distributed in accordance with the Creative Commons Attribution Non Commercial (CC BY-NC 4.0) license, which permits others to distribute, remix, adapt, build upon this work non-commercially, and license their derivative works on different terms, provided the original work is properly cited and the use is non-commercial. See: http://creativecommons.org/ licenses/by-nc/4.0/.

\section{Check for updates}

1 Cui D, Liu X, Hawkey P, et al. Use of and microbial resistance to antibiotics in China: a path to reducing antimicrobial resistance. J Int Med Res 2017;45:176878. doi:10.1177/0300060516686230

2 Lu Y, Hernandez P, Abegunde D, et al. The world medicines situation 2011. Medicine expenditures. World Health Organization,2011.

3 Holloway K, Van Dijk L. The world medicines situation 2011. Rational use of medicines. World Health Organization, 2011.

4 Mao W, Vu H, Xie Z, Chen W, Tang S. Systematic review on irrational use of medicines in China and Vietnam. PLoS One 2015;10:e0117710. doi:10.1371/journal.pone.0117710

5 Yin X, Song F, Gong Y, et al. A systematic review of antibiotic utilization in China. J Antimicrob Chemother 2013;68:2445-52. doi:10.1093/jac/dkt223

6 Wang M. Antimicrobial resistance in China: challenges and actions. Clin Infect Dis 2018;67(suppl_2):S127. doi:10.1093/cid/ciy702

7 Mao W, Chen W. The zero mark-up policy for essential medicines at primary level facilities. World Health Organization, 2015.

8 Liu GG, Vortherms SA, Hong X. China's health reform update. Annu Rev Public Health 2017;38:431-48. doi:10.1146/annurev-publhealth-031816-044247

9 Yang C, Shen Q, Cai W, et al. Impact of the zeromarkup drug policy on hospitalisation expenditure in western rural China: an interrupted time series analysis. Trop Med Int Health 2017;22:180-6. doi:10.1111/tmi.12817

10 Sun Q, Santoro MA, Meng Q, Liu C, Eggleston K. Pharmaceutical policy in China. Health Aff (Millwood) 2008;27:1042-50. doi:10.1377/hlthaff.27.4.1042

11 World Health Organization. Antimicrobial resistance: global report on surveillance 2014. WHO, 2014.

12 Meng Q, Mills A, Han Q. What can we learn from China's health system reform. BMJ 2019;365:12349. doi:10.1136/bmj.12349

13 Commission NHaFP. Status report on antimicrobial and antimicrobial resistance in China. Beijing Union Medical University Press, 2018

14 China MoHo. National special rectification activities program of clinical use of antibiotics (2011) 2011. http://www.moh.gov.cn/mohyzs/ s3586/201104/51376.shtml.

15 Xiao Y, Li L. Legislation of clinical antibiotic use in China. Lancet Infect Dis 2013;13:189-91. doi:10.1016/S1473-3099(13)70011-2

16 Dellit TH, Owens RC, McGowan JElr, et al, Infectious Diseases Society of America, Society for Healthcare Epidemiology of America. Guidelines for developing an institutional program to enhance antimicrobial stewardship. Clin Infect Dis 2007;44:159-77. doi:10.1086/510393

17 Yin J, Li Q, Sun Q. Antibiotic consumption in Shandong Province, China: an analysis of provincial pharmaceutical centralized bidding procurement data at public healthcare institutions, 2012-
16. J Antimicrob Chemother 2018;73:814-20. doi:10.1093/jac/dkx469

18 Xiao Y. Antimicrobial stewardship in China: systems, actions and future strategies. Clin Infect Dis 2018;67(suppl_2):S135-41. doi:10.1093/cid/ciy641

19 Fu H, Li L, Yip W. Intended and unintended impacts of price changes for drugs and medical services: Evidence from China. Soc Sci Med 2018;211:11422. doi:10.1016/j.socscimed.2018.06.007

20 Yin J, Wu C, Wei X, Sun Q. Antibiotic expenditure by public healthcare institutions in Shandong Province in China, 2012-2016. Frontiers in Pharmacology 2018;9:1396. doi:10.3389/ fphar.2018.01396

21 Qu X, Yin C, Sun X, et al. Consumption of antibiotics in Chinese public general tertiary hospitals (20112014): Trends, pattern changes and regional differences. PLoS One 2018;13:e0196668. doi:10.1371/journal.pone.0196668

22 Bao L, Peng R, Wang Y, et al. Significant reduction of antibiotic consumption and patients' costs after an action plan in China, 2010-2014. PLOS One 2015;10:e0118868. doi:10.1371/journal. pone.0118868

23 China NHaFPCotPsRo. The administration on the clinical use of antimicrobial agents and the status quo of antimicrobial resistance in China. Beijing Union Medical University Press, 2016.

24 Yang L, Liu C, Ferrier JA, Zhou W, Zhang X. The impact of the National Essential Medicines Policy on prescribing behaviours in primary care facilities in Hubei province of China. Health Policy and Planning 2013;28:750-60. doi:10.1093/heapol/czs116

25 Chen M, Wang L, Chen W, Zhang L, Jiang H, Mao W. Does economic incentive matter for rational use of medicine? China's experience from the essential medicines program. Pharmacoeconomics 2014;32:245-55. doi:10.1007/s40273-013-0068-z

26 Wei X, Yin J, Walley JD, et al. Impact of China's essential medicines scheme and zero-markup policy on antibiotic prescriptions in county hospitals: a mixed methods study. Trop Med Int Health 2017;22:1166-74. doi:10.1111/tmi.12922

27 Xie X, Jin X, Zhang L, et al. Trends analysis for drug utilization in county public hospitals: a sample study of the pilot area of health care reform in China. BMC Health Services Research 2018;18:812. doi:10.1186/s12913-018-3614-8

28 Tang Y, Liu C, Liu J, Zhang X, Zuo K. Effects of county public hospital reform on procurement costs and volume of antibiotics: a quasinatural experiment in Hubei Province, China. Pharmacoeconomics 2018;36:995-1004. doi:10.1007/s40273-018-0654-1

29 Ma X, Hong W, Li Y, Shi L, Liu X. Realigning the incentive system for China's primary healthcare providers. $B M$ 2019:365:12406. doi:10.1136/bmj.12406

30 Xue H, Shi Y, Huang L, et al. Diagnostic ability and inappropriate antibiotic prescriptions: a quasiexperimental study of primary care providers in rural China. J Antimicrob Chemother 2019;74:256-63. doi:10.1093/jac/dky390

31 Fletcher-Lartey S, Yee M, Gaarslev C, Khan R. Why do general practitioners prescribe antibiotics for upper respiratory tract infections to meet patient expectations: a mixed methods study. BMJ Open 2016;6:e012244. doi:10.1136/ bmjopen-2016-012244

32 Sun X, Jackson S, Carmichael GA, Sleigh AC. Prescribing behaviour of village doctors under China's New Cooperative Medical Scheme. Soc Sci Med 2009;68:1775-9. doi:10.1016/j. socscimed.2009.02.043

33 World Health Organization. Promoting rational use of medicines: core components. 2002. http://apps who.int/medicinedocs/pdf/h3011e/h3011e.pdf.

Cite this as: BMJ 2019;365:14016

http://dx.doi.org/10.1136/bmj.l4016 